\title{
Heterogeneity Does Matter for Tumor Characterization
}

\author{
Won Woo Lee ${ }^{1,2}$
}

Received: 29 April 2018 / Accepted: 4 May 2018 / Published online: 18 May 2018

(C) Korean Society of Nuclear Medicine 2018

\section{Heterogeneity Does Matter for Tumor Characterization}

Heterogeneity is welcomed by sociologists because diversity is considered a virtue for an open dynamic society. As for tumor characterization, heterogeneity seems to be a stigma that is highly associated with aggressiveness of the tumor, ultimately leading to poorer outcome of patients. The textural features of radiologic imaging studies, such as computed tomography (CT) or magnetic resonance imaging (MRI), have been studied as imaging biomarkers for tumor characterization [1], and a new discipline has emerged called "radiomics" [2]. The imaging features have evolved to be correlated with gene profiling, yielding the so-called "radiogenomics", which is reminiscent of a series of -omics in biology: genomics, proteomics, transcriptomics, epitranscriptomics, etc. Those omics studies are typically characterized by a huge amount of data, and effective data handling is a new challenge for biologists. Oncological positron emission tomography/ computed tomography (PET/CT) has also been intensively investigated for tumor characterization using ${ }^{18} \mathrm{~F}-\mathrm{FDG}\left({ }^{18} \mathrm{~F}\right.$ fluorodeoxyglucose) in most cases. The results of the recent radiomics approach of ${ }^{18} \mathrm{~F}-\mathrm{FDG} \mathrm{PET} / \mathrm{CT}$ are thoroughly reviewed in the current issue by Lee [3]. There are so many technical hurdles like protocol harmonization, target volume determination, and a time-consuming analysis process that implementation of the PET/CT technology does not seem easy. However, a number of papers as introduced in the review paper may render readers to rethink the feasibility of the tumor

Won Woo Lee

wwlee@snu.ac.kr

1 Department of Nuclear Medicine, Seoul National University Bundang Hospital, Seoul National University College of Medicine, 82, Gumi-ro 173 Beon-gil, Bundang-gu, Seongnam-si, Gyeonggi-do 13620, Korea

2 Institute of Radiation Medicine, Medical Research Center, Seoul National University, Seoul, South Korea heterogeneity analysis as an imaging biomarker for prediction of patients' outcome.

When it comes to the prognosis prediction of oncologic patients, some quantitative parameters have been widely employed. Standardized uptake value (SUV), metabolic tumor volume (MTV), and total lesion glycolysis (TLG) are the famous trio with an abundance of corresponding papers in the literature of the past decades. Conceptionaly, the ${ }^{18} \mathrm{~F}$ FDG PET/CT radomics approach includes the three parameters and other texture analysis-driven parameters (skewness, kurtosis, etc). Therefore, an increase in the number of parameters is a sticking point. In contrast to the big data analysis in biology, clinical data analysis in medicine usually favors facility. That is one of the reasons why the simplest SUVmax (maximum standardized uptake value) has been mainly advocated among other parameters in the daily routine report of oncologic ${ }^{18} \mathrm{~F}-\mathrm{FDG}$ PET/CT studies.

The recent trend of texture analysis in nuclear medicine may reflect the potential of the radiomics approach, and the tumor heterogeneity may be well represented by the analysis. However, it remains to be seen whether the efforts and enthusiasm of nuclear medicine physicians for radiomics will be paid off by improved patients' outcome.

\section{Compliance with Ethical Standards}

Conflicts of Interest The author declare that they have no conflict of interest.

\section{References}

1. Lee G, Bak SH, Lee HY. CT radiomics in thoracic oncology: technique and clinical applications. Nucl Med Mol Imaging. 2018;52: 91-8.

2. Park JE, Kim HS. Radiomics as a quantitative imaging biomarker: practical considerations and the current standpoint in neurooncologic studies. Nucl Med Mol Imaging. 2018;52:99-108.

3. Lee JW, Lee SM. Radiomics in oncological PET/CT: clinical applications. Nucl Med Mol Imaging. 2017. https://doi.org/10.1007/ s13139-017-0500-y 